\title{
Formal Verification of Orchestration Templates for Reliable Deployment with OpenStack Heat*
}

\author{
$1^{\text {st }}$ Adja Ndeye Sylla \\ Orange Labs, \\ Rennes, France \\ adjandeye.sylla@orange.com \\ $5^{\text {th }}$ Philippe Merle \\ Spirals project-team, \\ Inria Lille - Nord Europe, \\ Lille, France \\ philippe.merle@inria.fr
}

\author{
$2^{\text {nd }}$ Karine Guillouard \\ Orange Labs, \\ Rennes, France \\ karine.guillouard@orange.com
}

\author{
$3^{\text {rd }}$ Frédéric Klamm \\ Orange Labs, \\ Rennes, France
}

\author{
$4^{\text {th }}$ Meryem Ouzzif \\ Orange Labs, \\ Rennes, France
}

frederic.klamm@orange.com

\author{
$6^{\text {th }}$ Souha Ben Rayana \\ Univ. Grenoble-Alpes, INRIA, \\ CNRS, Grenoble INP, LIG, \\ Grenoble, France \\ souha.ben-rayana@inria.fr
}

\author{
$7^{\text {th }}$ Jean-Bernard Stefani \\ Univ. Grenoble-Alpes, INRIA, \\ CNRS, Grenoble INP, LIG, \\ Grenoble, France \\ jean-bernard.stefani@inria.fr
}

\begin{abstract}
In the context of Network Functions Virtualization (NFV), telecommunication systems are more and more deployed on the cloud, using orchestration engines such as OpenStack Heat. Heat takes as input templates that describe the components of the target system and automatically performs the deployment. This prevents consumers of cloud services from handling the challenges of manual deployment. However, deploying such systems remains challenging. Indeed, the templates given to Heat may contain errors that can lead to a failed or a partial deployment, thus compromising the systems reliability. To handle this challenge, we propose a formal approach and a tool for the verification of templates consistency prior to launching their deployment. A case study is presented to validate the approach.

Index Terms-Formal verification, OpenStack, Heat, NFV
\end{abstract}

\section{INTRODUCTION}

Today's systems, in both IT (e.g., smart buildings and cities) and Telecommunication (e.g., 5G [1], solutions that rely on Network Functions Virtualization (NFV) [2] [3]) domains, consist of many heterogeneous and interacting components. These systems are more and more deployed using cloud technologies, especially to increase their performance, scalability, and accessibility. Examples of cloud management technologies are Microsoft Azure [4], IBM Cloud [5], OpenNebula [6], and OpenStack [7]. OpenStack is among the most used technologies in the NFV context [8] [9]. It is open-source, and has a modular architecture. It also provides an orchestration engine, named Heat [10], for the automatic deployment of systems.

Heat takes as input a template that describes the components of the target system and automatically performs the deployment. This allows consumers of cloud services to not handle the challenges of a manual deployment. Indeed, they just have to provide Heat with the template of the target system. This template can be hand-written or even generated by a tool. However, deploying such a system remains challenging. Indeed, the templates given to Heat may contain errors that, if

* This work is funded by Orange Labs in the context of the $<\mathrm{I} / \mathrm{O} \mathrm{Lab}\rangle$. The authors thank the IRT $\mathrm{b}<>$ com members who provided the source code for the UGW use-case, particularly Emmanuel Gouleau and Philippe Bertin. not detected early, can lead to a failed or partial and useless deployment. This causes waste of resources, and reliability issues because one may wrongly assume that the deployment succeeded. In addition, consumers of cloud services may lose a lot of time when deploying an erroneous template. Indeed, they launch their deployment and notice after a delay, that can be long, that the deployment failed. In this case, they have to manually try to detect the error. This can also take a lot of time, and may be not trivial especially for large templates.

To solve the challenges of reliable deployments with Heat, we propose a formal approach for the verification of templates consistency. This verification is done prior to launching the deployment and allows the detection of different types of errors: syntax, type and consistency. Our contributions are:

1) a review of the HOT specification [11] [12] (language used to design Heat templates) and its formalization;

2) the development of a tool that allows consumers of cloud services to verify their templates before deployment;

3) a case study in the NFV context to validate our approach.

This paper is structured as follows. Section II first discusses related work. Then, Section III gives the background notions. Section IV presents the review of the HOT specification and its formalization. Section $\mathrm{V}$ describes the implemented tool. Section VI presents a case study in the NFV context. Finally, Section VII concludes the paper and gives future directions.

\section{RELATED WORK}

Several approaches, based on formal methods, have been proposed for the reliability of deployments in the cloud [13].

In [14], the authors target the NFV domain and propose a solution to both compute VNFs placement and verify if reachability policies (e.g., isolation, latency) can be met before deployment. The VNFs placement and the reachability problem are formally modeled as clauses resolved by a solver. However, this approach does not enable templates verification.

Heat provides a set of tools for the verification of templates before deployment. These tools consist of the Heat parser, the 
heat template-validate command, and the dry-run option [15]. These tools perform syntax and type checking on a template. However, using them requires users to connect to a running OpenStack platform even if their aim is to just verify the template without deploying it. Moreover, these tools do not detect errors that are related to the consistency of templates.

\section{BACKGROUND}

This section first introduces HOT. Then, it presents Location Graphs and Alloy which are used in the proposed approach.

\section{A. HOT}

HOT [11] is a YAML-based language used by Heat. This language allows to describe a system as a set of resources of different types (e.g., virtual machines, physical servers, networks and ports). These resources are defined in a template.

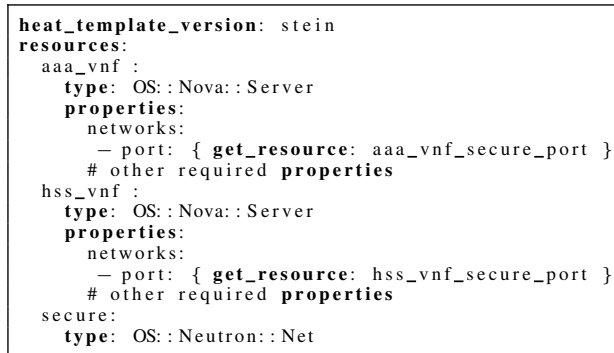

Listing 1. Example of Heat template

Listing 1 presents an example of Heat template. This template is written with the latest version of HOT (stein). It describes a system that consists of two virtual machines (aaa_vnf, hss_vnf), and one network (secure). Each machine has a port that connects it to the network (cf. lines 7 and 13).

HOT relies on a specification that defines its core concepts [11] and the supported resource types [12]. This specification allows consumers of cloud services to know how to design templates that can be automatically deployed with Heat. However this specification is mostly written in natural language and is therefore, possibly ambiguous, inconsistent, and error-prone. Hence, based on this specification, users may design erroneous and inconsistent Heat templates. To prevent this, we decide to review and formalize the HOT specification.

\section{B. Location Graphs}

Location Graphs (LG) [16] [17] is a formal framework for modeling heterogeneous and distributed component-based systems. It provides us with a reference meta-model for the formal modeling of cloud configuration languages. In this paper, LG is used to formally model the HOT specification.

A Location Graph is a set of Locations. Each Location is a locus of concurrent computations as in process calculi with localities such as the distributed $\pi$-calculus [18] or kell calculi [19]. From a software engineering point of view, a Location can be seen as a component or as a connector, as in component-based models [20]. Each Location has a Name for its identification, a Sort and a Process respectively for its encapsulation and its behavior, as well as a set of required and provided Roles. Roles correspond to the points of attachment of a Location, in order to enable its interaction with other Locations. Location Names, Sorts, Processes, and Roles are all instances of Value.

A Location Graph has four invariants: (1) Locations are uniquely named, (2) the required and provided Roles of a Location are disjoint, (3) a Role is provided by a single Location, (4) a Role is required by a single Location. The Location Graphs concepts are implemented in Alloy.

\section{Alloy}

Alloy [21] provides a lightweight formal language and an automated analyzer to specify and verify systems. It is chosen because it is widely known, in the formal methods community, open source, and accessible as its language has few concepts.

The Alloy language is based on a first order relational logic [22]. Its concepts include signature with fields for describing the components of the target system and their interactions, and fact for defining a set of invariants of the system. It also provides commands for validating the system.

An example of such command is run. When executed, this command triggers the Alloy Analyzer which finds one or several instances of the system, as a constraint satisfaction problem resolved by a solver, if its model is consistent (i.e. all constraints are satisfiable). When the model is inconsistent, the analyzer highlights the set of constraints that cannot be satisfied. When an instance is found, it can be visualized graphically and/or analyzed in order to verify its properties.

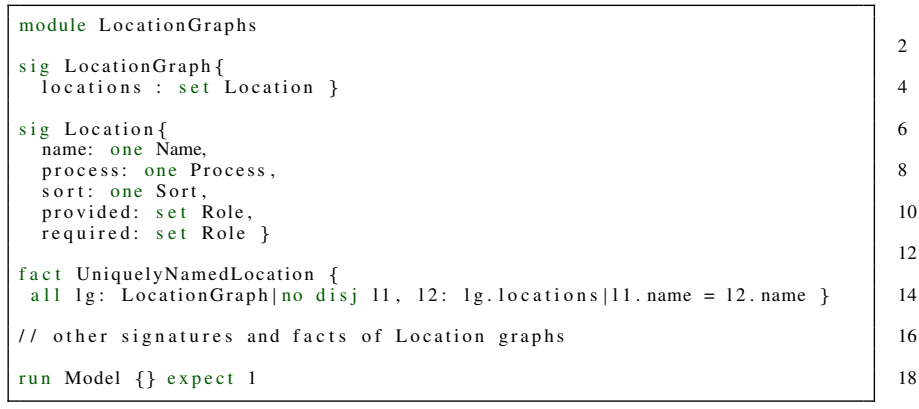

Listing 2. Alloy implementation of Location graphs

Listing 2 shows the implementation of Location Graphs as a module in Alloy. A Locationgraph is a signature (sig) with a set of Locations (lines 3-4). A Location is a signature with one mandatory Name, Process, and Sort (lines 7-9). A Location also has provided and required Roles (lines 10-11). Lines 13-14 show the UniquelyNamedLocation invariant specifying that distinct Locations of a Locationgraph must have different Names. This module has a run command to verify LocationGraph and find instances, if there is at least one.

Fig. 1 shows an instance of Locationgraph found by the Alloy Analyzer, after the execution of the run command (line 18 in Listing 2). This instance has two Locations, two Names, one Process, and one Sort. Note that the Locations have distinct Names to satisfy the UniquelyNamedLocation invariant. Processes and Sorts could be shared between Locations, as in Fig. 1. 


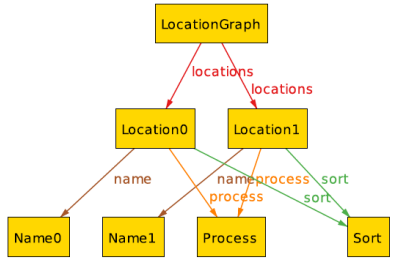

Fig. 1. LocationGraph instance found by Alloy Analyzer

\section{REVIEW AND FoRMALIZATION OF THE HOT SPECIFICATION}

This section first describes the HOT specification. Then, it shows how this specification is reviewed and then formalized.

\section{A. Description of the HOT Specification}

The paper targets the latest HOT specification (stein). The HOT core specification states that a template has 7 sections:

- heat_template_version: defines the version of HOT that is used to design the template. This section is mandatory;

- description: is an optional string to explain the template;

- parameters: optionally defines the input parameters that have to be provided at deployment time. This allows to customize the template and reuse it in different contexts.

- parameter_groups: specifies how the input parameters are grouped. For example, parameters related to network (e.g., IP addresses, CIDR) can belong to the same group;

- resources: defines a set of resource types to be deployed;

- outputs: optionally defines the outputs to give a feedback to users after the deployment (e.g. dynamic IP address);

- conditions: optionally defines a set of conditions, to be evaluated, based on the values of the input parameters.

The HOT types specification [12] is also written in natural language but its YAML version can be retrieved from a running OpenStack platform. This YAML version is readable by machines and can, therefore, be used for generation purposes. This specification defines the supported resource types. These types are provided by the OpenStack services and are grouped in domains (e.g., computing, networking). For instance, the Nova service provides computing resource types. An example of these types is OS::Nova::Server (virtual machine). The Neutron service provides networking resource types such as OS::Neutron::Net and OS::Neutron::Port. Each resource type has a support status, a set of properties, and a set of attributes.

\section{B. Review and Formalization of the HOT Specification}

The aim of reviewing the HOT specification is to verify its consistency and its correctness. To perform the review, we (1) formally model the HOT specification with Location Graphs and Alloy, (2) find instances of the obtained model with the Alloy Analyzer and analyze the instances. This allows us to detect about 63 errors in the HOT specification. These errors are related to missing invariants that prevent inconsistent behaviors. In the following, the modeling of the HOT specification is first presented. Then, two examples of errors are given. Finally, the formalization of the HOT specification, to correct the detected errors, is shown.

1) Modeling the HOT specification: the HOT core is first modeled by writing, systematically from the specification, an Alloy module named HOT.als. This is done by defining an inheritance relationship and a mapping between the HOT core concepts and those of Location Graphs. A Template inherits from LocationGraph as both can be seen as a componentbased system. Resource inherits from Location, both can be seen as a system component. Parameter_group, Parameter, Output, and Condition inherit from Value. A run command is written to find Template instances. Then, the model of the HOT types specification is generated by a dedicated Python script. It takes as input the YAML version of the HOT types specification and generates an Alloy module named HOT_types.als. This module includes, for each resource type, a set of signatures, and a run command to find its instances.

2) Examples of detected errors: let us consider, in Fig. 2, an instance of HOT template found by the Alloy Analyzer. In this instance, a network has two subnets with the same address (10.0.0.0/24). This behavior is inconsistent. However, this instance is found by the analyzer. The reason is that there is, in the HOT specification, no invariant that prevents this.

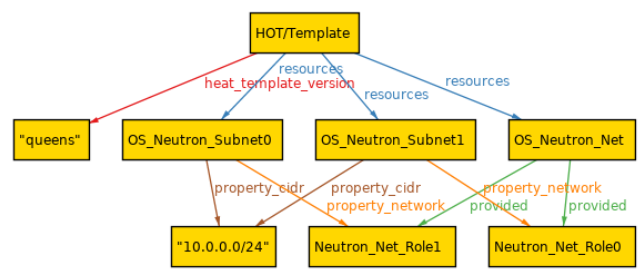

Fig. 2. Two subnets with the same address in the same network

Fig. 3 shows another instance found by the analyzer. This instance consists of 2 virtual machines and 1 port attached to both machines (the port has 2 provided roles and each of them is attached to one of the virtual machines). This behavior is inconsistent and, thus, it must be avoided through an invariant.

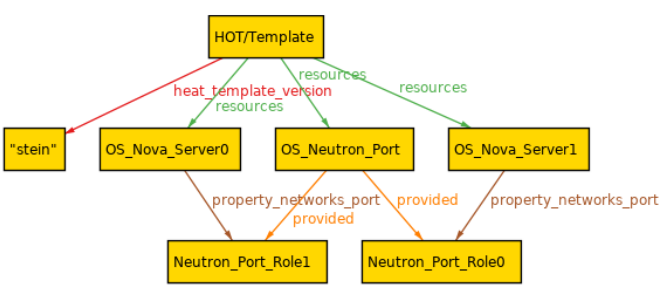

Fig. 3. Two virtual machines with the same port

3) Formalizing the HOT specification: the aim is to correct the errors detected in the HOT specification, by taking into account the missing invariants. This formalization is done by writing in Alloy a fact for each detected error. These facts are added in the HOT.als (resp. HOT_types.als) module for the errors related to HOT core (resp. supported resource types). 
For instance, Listing 3 shows the Alloy fact written to correct the error presented in Fig. 3. This fact states that distinct resources of type OS::Nova::Server must have different ports.

fact no_distinct_servers_with_the_same_port \{

all disj s1, s2: OS_Nova_Server|\#(s1.property_networks_port. " provided \& s2. property_networks_port. ${ }^{\sim}$ provided) $=0$

Listing 3. Fact for OS::Nova::Server and OS::Neutron::Port

\section{IMPLEMENTED TEMPLATES VERIFICATION TOOL}

This tool takes as input a Heat template with its environment file, if there is one. This file defines the external resources that are used in the template and gives values to the parameters.

The tool is based on the formalized HOT specification and on the Alloy Analyzer. It consists of (1) a parser that performs syntax and type checking, and (2) a generator that translates the template and its environment file into a formal Alloy model. This model consists of a signature, a fact, and a run command. The signature declares the parameters, parameter_groups, resources, outputs and conditions sections of the template. The fact affects values to the fields of the sections. The run command is executed by the Alloy Analyzer, to check if the template is compliant with the formalized HOT specification (i.e. the template does not contain errors and can be deployed). If the template is deployable, an instance of it is found. Otherwise, its list of errors is returned to the user.

\section{CASE Study}

To validate our approach, we target a virtual network service developed by the IRT $b<>$ com. This network service (cf. Fig. 4), called Unifier Gateway (UGW) [23], is a convergent access control solution based on NFV technologies. It consists of a user and a control planes. The latter is made up of 6 VNFs:

- AAA: Authentication, Authorisation \& Accounting;

- DHCP: Dynamic Host Control Protocol;

- HSS: Home Subscriber Server;

- MME: Mobility Management Entity;

- S/PGW-C: Serving \& PDN Gateway Control plane;

- GW-U: SDN Controller (SDN-Ctlr) of the user plane.

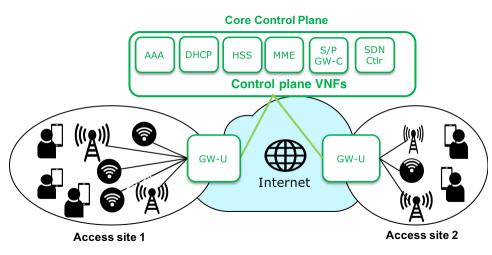

Fig. 4. UGW network service

For the UGW rolling up, $\mathrm{b}<>$ com provides a Heat template in order to deploy the control plane on an OpenStack platform.

\section{A. Heat Template of the UGW}

This template defines 5 private networks (e.g., secure, lte control) and 1 public network. It also defines 7 Virtual Machines (VM) on which specific services are installed. There is one VM for each VNF and another one called Internal switch for routing the network traffic by applying specific rules. Each
$\mathrm{VM}$ is connected to a set of private networks through ports. An extract of this template is the example given in Listing 1.

\section{B. Verification of the UGW Template}

We give the UGW template, and its environment file, to our tool for its verification. Then, the Alloy Analyzer is able to find a UGW instance. This means that there is no error in the template. We expected this result because the template has been used to deploy, on several sites, an operational UGW.

Therefore, to better illustrate our approach, we introduce an error in the UGW template (cf. Listing 1) by changing the port of the aaa_vnf, on the secure network, to hss_vnf_secure_port. After this, hss_vnf_secure_port is attached to both machines.

Then, we deploy the new template. Heat does not detect the error and starts the deployment which takes 5 minutes to end. When checking the deployment state, we notice that it is partially done (cf. Fig. 5). Indeed, hss_vnf_secure_port cannot be attached to both machines. For a larger system, the deployment may take a longer time without being successful.

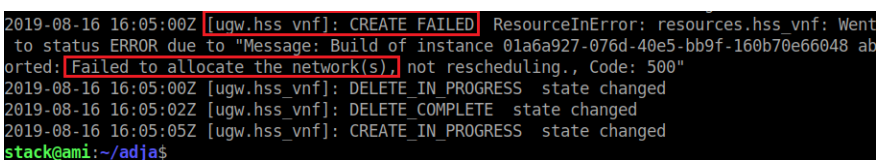

Fig. 5. Deployment of the modified UGW template

Finally, we verify the modified template with our tool to see its behavior. In this case, as shown in Fig. 6, the error is detected, in $876 \mathrm{~ms}$, before starting the deployment. Indeed, no instance of UGW is found and the tool highlights the fact that it cannot satisfy. This fact is the example given in Listing 3 .

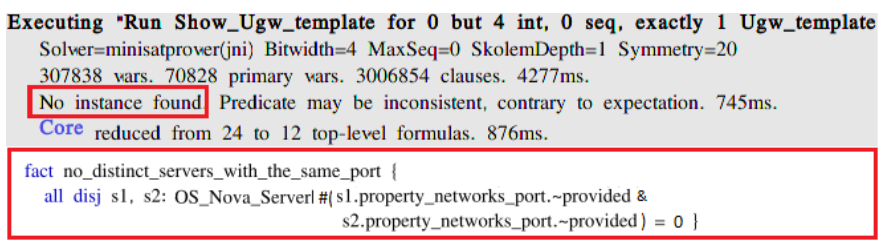

Fig. 6. Formal verification the modified UGW template

\section{CONCLUSION}

This paper has proposed a formal approach to improve the reliability of HOT templates deployment through the Heat engine. This is done by (1) reviewing the HOT specification and identifying a set of errors it contains (missing invariants to prevent inconsistent behaviors), (2) formalizing the HOT specification to correct these errors, and (3) implementing a tool that verifies templates consistency, based on the formalized HOT specification, prior to launching their deployment.

A first perspective of this work is to consider more types of resources. Indeed, in the current status, only 36 types are considered. Another perspective is to ensure the evolution of this approach, as well as the implemented verification tool, in order to support the future versions of HOT. Finally, a major perspective is to generalize this approach to other orchestration languages such as Cloudify [24], and Docker Compose [25]. 


\section{REFERENCES}

[1] A. Manzalini, C. Lin, J. Huang, C. Buyukkoc, M. Bursell et al., "Towards 5G Software-Defined Ecosystems: Technical Challenges, Business Sustainability and Policy Issues," IEEE SDN White Paper, 2016.

[2] B. Han, V. Gopalakrishnan, L. Ji, and S. Lee, "Network Function Virtualization: Challenges and Opportunities for Innovations," IEEE Communications Magazine, vol. 53, no. 2, pp. 90-97, 2015.

[3] R. Mijumbi, J. Serrat, J. L. Gorricho, N. Bouten, F. De Turck, and R. Boutaba, "Network Function Virtualization: State-of-the-art and Research Challenges," IEEE Communications Surveys \& Tutorials, vol. 18, no. 1 , pp. 236-262, 2015.

[4] "Microsoft Azure," accessed: 2019-08-14. [Online]. Available: https: //azure.microsoft.com/en-us/

[5] "IBM Cloud," accessed: 2019-09-16. [Online]. Available: https: //www.ibm.com/cloud

[6] "OpenNebula," accessed: 2019-08-14. [Online]. Available: https: //opennebula.org/

[7] "OpenStack," accessed: 2019-08-14. [Online]. Available: https://www. openstack.org/

[8] F. Callegati, W. Cerroni, C. Contoli, and G. Santandrea, "Performance of network virtualization in cloud computing infrastructures: The openstack case," in 2014 IEEE 3rd International Conference on Cloud Networking (CloudNet). IEEE, 2014, pp. 132-137.

[9] F. Foresta, W. Cerroni, L. Foschini, G. Davoli, C. Contoli, A. Corradi, and F. Callegati, "Improving openstack networking: Advantages and performance of native sdn integration," in 2018 IEEE International Conference on Communications (ICC). IEEE, 2018, pp. 1-6.

[10] "Heat: Openstack orchestration engine," accessed: 2019-08-14. [Online]. Available: https://wiki.openstack.org/wiki/Heat

[11] "HOT Core Specification," accessed: 2019-08-14. [Online]. Available: https://docs.openstack.org/heat/stein/template_guide/hot_spec.html

[12] "HOT Types Specification," accessed: 2019-08-14. [Online]. Available: https://docs.openstack.org/heat/stein/template_guide/openstack.html

[13] A. Souri, N. J. Navimipour, and A. M. Rahmani, "Formal Verification Approaches and Standards in the Cloud Computing: A Comprehensive and Systematic Review," Computer Standards \& Interfaces, vol. 58, pp. $1-22,2018$

[14] G. Marchetto, R. Sisto, J. Yusupov, and A. Ksentini, "Virtual Network Embedding with Formal Reachability Assurance," in 2018 14th International Conference on Network and Service Management (CNSM). IEEE, 2018 , pp. $368-372$.

[15] "Heat validation tools: information about the dry-run option," accessed: 2019-08-14. [Online]. Available: https://docs.openstack.org/newton/ user-guide/cli-create-and-manage-stacks.html

[16] J. B. Stefani, "Components as Location Graphs," in International Conference on Formal Aspects of Component Software. Springer, 2014, pp. 3-23.

[17] J. Stefani and M. Vassor, "Encapsulation and Sharing in Dynamic Software Architectures: The Hypercell Framework," in 39th IFIP Int. Conf. Formal Techniques for Distributed Objects, Components, and Systems (FORTE), ser. Lecture Notes in Computer Science, vol. 11535. Springer, 2019.

[18] M. Hennessy, J. Rathke, and N. Yoshida, "SafeDPi: A Language for Controlling Mobile Code," in International Conference on Foundations of Software Science and Computation Structures. Springer, 2004, pp. 241-256.

[19] A. Schmitt and J. B. Stefani, "The Kell Calculus: A Family of HigherOrder Distributed Process Calculi," in International Workshop on Global Computing. Springer, 2004, pp. 146-178.

[20] I. Crnkovic, S. Sentilles, A. Vulgarakis, and M. R. V. Chaudron, "A Classification Framework for Software Component Models," IEEE Transactions on Software Engineering, vol. 37, no. 5, pp. 593-615, 2010.

[21] D. Jackson, Software Abstractions: Logic, Language, and Analysis. MIT press, 2012.

[22] — , "Automating first-order relational logic," in ACM SIGSOFT Software Engineering Notes, vol. 25, no. 6. ACM, 2000, pp. 130-139.

[23] "b $<>$ com, Wireless Edge Factory," https://b-com.com/en/ bcom-wireless-edge-factory, 2019.

[24] "Cloudify," accessed: 2019-08-23. [Online]. Available: https://cloudify. $\mathrm{co} /$

[25] "Docker Compose," accessed: 2019-08-16. [Online]. Available: https: //docs.docker.com/compose/ 